maturing network of enteric glia regulates the epithelial barrier, so we aim to show rescue is due to replacement of glial factors. METHODS/STUDY POPULATION: Jejunal tissues from suckling or weaned pigs were assessed by RNAseq and processed for immunofluorescent histology and 3-D volume imaging. Jejunal ischemia was surgically induced in weaned pigs and injured mucosa was recovered ex vivo with or without the glial inhibitor fluoroacetate (FA) while monitoring transepithelial electrical resistance (TER). RESULTS/ ANTICIPATED RESULTS: Ingenuity Pathways Analysis of RNAseq data revealed significant suppression of numerous pathways critical for epithelial wound healing in suckling pigs ( $Z$-score $<-2$ for of nine key pathways). Volume imaging studies confirmed lower density $(\mathrm{P} \leq 0.05)$ and complexity of the subepithelial glial network in suckling pigs. Treatment with FA inhibited TER recovery $(\mathrm{P}<0.0001)$ and restitution $(\mathrm{P}<0.05)$ in weaned pigs, mimicking the suckling pig phenotype and supporting glia as an important regulator of restitution in our model. DISCUSSION/SIGNIFICANCE OF IMPACT: These findings provide important evidence that a developing glial network may be critical to the postnatal development of intestinal barrier repair mechanisms. Ongoing work will explore glial-epithelial interactions in vitro to further define postnatal development of barrier repair.

4581

\author{
Autonomic Dysfunction as a Marker of Depression and \\ Coronary Artery Disease \\ Anish Sanjay Shah ${ }^{1}$ \\ ${ }^{1}$ Emory University
}

OBJECTIVES/GOALS: Dysfunction of the autonomic nervous system (ANS) may be important in both depression and coronary artery disease (CAD). A novel heart rate variability (HRV) metric, Dyx, may be a be a potentially useful tool to study ANS dysfunction in these diseases. We propose that ANS dysfunction, measured by decreased Dyx, will associate with both depression and obstructive CAD. METHODS/STUDY POPULATION: We included participants undergoing coronary angiography for suspected CAD. Depressive symptoms were assessed with the Patient Health Questionnaire-9 (PHQ-9). HRV data were collected continuously on participants before catheterization using a new ECG patch (VivaLNK). We assessed HRV by Dyx (primary) and high and low frequency power, multiscale entropy, and deceleration capacity. Two-sample t-tests and logistic regressions (with adjustment for age and sex) were used to study the difference in HRV (before cardiac catheterization) between those with high versus low depressive burden (PHQ-9 $\geq 10$ ), and in those with versus without obstructive CAD (>70\% stenosis). RESULTS/ANTICIPATED RESULTS: We assessed 30 individuals with mean (SD) age 62.4 (13.2); 7.1\% were female and $15.4 \%$ were black. Mean Dyx in high depressive symptoms $(\mathrm{N}=21,70 \%)$ was $1.8(0.2)$ and in none-low depressive symptoms $(\mathrm{N}=7,23 \%)$ was $2.2(0.2)$. Differences were also observed for high frequency (HF) $(4.4(1.1)$ vs. $6.0(1.4))$ and deceleration capacity $(-4.2(2.1)$ vs. $-10.7(8.5))$. Mean Dyx in obstructive CAD $(\mathrm{N}=17$, $57 \%)$ and non-obstructive $\mathrm{CAD}(\mathrm{N}=10,33 \%)$ was $1.7(0.6)$ and 2.6 (1.2) respectively. Differences were seen with sample entropy (1.2 (0.2) vs. $1.5(0.2)$ ). Every 1 unit of $\log (\mathrm{HF})$ had an odds ratio $=0.14(95 \%$ CI $0.06-0.36)$ for depression. DISCUSSION/ SIGNIFICANCE OF IMPACT: ANS dysfunction, measured by $\mathrm{HRV}$, associates with both depression and obstructive CAD.
Autonomic ECG markers may play an important role in assessing brain-heart pathology, and may be useful to study the interaction between depression and CAD.

4511

Bio-Compatible Implantable Oxygen Sensor Technology with Real-Time Monitoring of Surgical Flaps and Reimplantation

Preet Patel, Patel $^{1}$, Mohamed Ibrahim, and Bruce Klitzman ${ }^{2}$

${ }^{1}$ Duke University; ${ }^{2}$ Dept of Plastic and Reconstructive Surgery, Duke University

OBJECTIVES/GOALS: Current surgical flap and replantation monitoring techniques have limitations in detecting the pathologic state, calibration and cost-to-patient issues. Our hypothesis is that novel implantable oxygen sensors can provide a more efficient, accurate, and reliable monitoring of tissue oxygenation. METHODS/ STUDY POPULATION: Experimental sensors were used with an exogenous remote used as a reader once implanted (Fig. 1) A rat tissue perfusion model with three regions of an SIEA flap as well as into adjacent control sites was made (Tip, Middle, and Base) Blood flow was greatest at the base, diminishing towards the Tip, thus creating a perfusion gradient. Changes in tissue oxygen tension $\mathrm{PO} 2$ were estimated by the steady-state fluorescence of the optical sensors using an IVIS imaging system. The sensors were used to collect data from days 0,3 , and 7 as a reading of Tissue Oxygen Tension (TOT) with ANOVA used to assess for statistical significance in blood oxygen data with respect to relative perfusion status. RESULTS/ ANTICIPATED RESULTS: Inspired FiO2 was decreased from $100 \%$ to $12 \%$ with a corresponding change in the TOT readings from all sensors. (Fig. 2) The tip portion of the flap demonstrated the most profound detection of tissue necrosis, with the middle demonstrating the second most necrosis and the base demonstrating the least with correlating TOT sensor readings. (Fig. 3) Acute vascular compromise of the feeding blood vessels in the pedicle was immediately detected within 70 seconds $(* \mathrm{p}<0.05)$. (Fig. 4) DISCUSSION/ SIGNIFICANCE OF IMPACT: This study introduces and validates a recent technique to monitor acute vascular occlusion, flap viability, and necrosis in the immediate postoperative period in a validated rodent model. Future directions of this novel technology will aim to reproduce these findings in clinical feasibility studies.

4119

\section{Cholecystokinin (CCK) Receptor Antagonist Reverses Nonalcoholic Steatohepatitis (NASH) by Reducing Hepatic Macrophages and Inflammatory Cytokines ${ }^{\dagger}$ Martha Gay ${ }^{1}$, Anita Safronenka², Hong Cao ${ }^{2}$, Robin Tucker ${ }^{2}$, Narayan Shivapurkar ${ }^{2}$, Annie Kruger ${ }^{2}$, and Jill Smith² ${ }^{1}$ Georgetown - Howard Universities; ${ }^{2}$ Georgetown University}

OBJECTIVES/GOALS: NASH increases the risk of cirrhosis and liver cancer. High-fat diets increase CCK levels and CCK receptors have been identified on fibroblasts and immune cells. We hypothesized that CCK receptor blockade could prevent NASH by altering the hepatic microenvironment and macrophage activation. METHODS/STUDY POPULATION: Female mice were fed a Choline Deficient Ethionine supplemented (CDE) saturated fat diet or control high-fat diet for 18 weeks. Mice in each group were treated with a CCK receptor antagonist, proglumide $(0.1 \mathrm{mg} / \mathrm{ml})$ in the 
drinking water or regular water. Resected livers were stained for $\mathrm{H} \& \mathrm{E}$ for features of NASH and F4/80 for macrophages analysis. Liver RNA was evaluated for the expression of cytokines and chemokines using an 84-gene Profiler array (Qiagen). Oxidative stress was analyzed by qRT-PCR for heat shock proteins (HSPs) 27, 60, 70 and 90 and for glutathione by a fluorometric assay. Differences in CDE fed and CDE/proglumide-treated mouse livers were evaluated. RESULTS/ANTICIPATED RESULTS: Livers from mice on the $\mathrm{CDE}$ diet displayed histologic features of NASH that were prevented by proglumide. Cytokines and chemokines expression, especially CCL20 and CCL2, were increased in the CDE fed mice and these levels were reduced greater than 20 -fold with proglumide. Infiltration of $\mathrm{F} 4 / 80+$ macrophages was markedly increased in the CDE livers and these were reduced by $>50 \%(\mathrm{p}<0.0001)$ with proglumide. RNA expression of HSP70 $(p=0.006)$ and HSP27 $(p=0.011)$ were reduced with proglumide. Hepatic glutathione concentration more than doubled in the $\mathrm{CDE} /$ proglumide treated mice compared to CDE mice. CCK-B receptor expression increased in the CDE-fed mouse livers compared to controls. DISCUSSION/ SIGNIFICANCE OF IMPACT: CCK receptor blockade decreases NASH by reducing hepatic macrophages, oxidative stress, and blocking inflammatory cytokines and chemokines. This data supports our novel hypothesis that CCK receptors play a role in NASH and proglumide may provide an innovative treatment for this condition.

\section{5}

Concurrent assessment of experimental pain and selfreported pain intensity with acute exercise intervention in fibromyalgia; clarifying or obscuring clinical outcomes?

Giovanni Berardi ${ }^{1}$, Grace Ptizen ${ }^{1}$, Matthew Dellalacono ${ }^{1}$, and Marie Hoeger Bement ${ }^{1}$

${ }^{1}$ Marquette University

OBJECTIVES/GOALS: Experimental pain testing is used to identify changes in nociceptive processing and outcomes with intervention. This study investigated exercise induced changes in experimental pain and self-reported pain intensity after an acute bout of exercise in participants with fibromyalgia. METHODS/STUDY POPULATION: Ten females with fibromyalgia $(55 \pm 10 \mathrm{yr})$ were familiarized to study procedures and underwent submaximal $(20 \%$ maximal voluntary contraction) intermittent eccentric muscle contractions isolated to the right arm for 10-minutes. Self-reported pain intensity (0-10 numerical pain rating scale [NPRS]) of the exercising arm was measured before, during, and after exercise; whole-body pain intensity was measured before and after exercise. Experimental pain testing included measurement of pressure pain thresholds ( $\mathrm{kPa}$ [PPTs]); temporal summation (TS) of pressure pain with a constant mechanical pressure; and TS of punctate pressure with repeated application of monofilaments before and after exercise. RESULTS/ANTICIPATED RESULTS: Participants reported minimal to moderate arm pain $(3.1 \pm 2.1)$ during exercise. Following exercise, arm pain and whole-body pain significantly increased $(3.1 \pm 2.2$ and $1.6 \pm 0.5$, respectively) [ $\mathrm{p}<0.05$ ]. No change occurred with PPTs at the bicep (138.9 \pm 49.5 to $142.8 \pm 55.3)$, PPTs at the quad $(212.0$ \pm 105.4 to $228.1 \pm 100.0)$, TS of mechanical pressure pain $(7.6 \pm 2.1$ to $7.9 \pm 1.5)$, TS of punctate pressure pain at the bicep $(2.6 \pm 1.7$ to $3.0 \pm 1.5)$, and TS of punctate pressure pain at the quad (3.6 \pm 1.5 to $3.7 \pm 1.4)$ before to after exercise respectively $[p>0.05]$. The change in self-reported arm and whole-body pain did not correlate with the change in experimental pain testing. DISCUSSION/ SIGNIFICANCE OF IMPACT: In people with fibromyalgia, there is no relation between self-reported clinical pain and experimental pain following a single exercise session. Further research should identify the influence of exercise training on pain perception and if experimental pain testing translates to clinical insight.

\section{Decreased structural basal ganglia motor loop connections in Vascular Parkinsonism compared to Parkinson's disease and healthy aging}

Christine Cooper ${ }^{1}$, Federico Rodriguez-Porcel ${ }^{1}$, Travis Turner ${ }^{1}$, Gonzalo Revuelta ${ }^{1}$, Jens Jensen ${ }^{1}$, Vanessa Hinson ${ }^{1}$, and Leonardo Bonilha $^{1}$

${ }^{1}$ Medical University of South Carolina

OBJECTIVES/GOALS: This study uses diffusion kurtosis imaging (DKI) to investigate the structural profiles of basal ganglia (BG) motor circuitry in Vascular Parkinsonism (VP), Parkinson's disease (PD), and healthy aging controls (HC). VP is a clinical diagnosis of lower body predominant parkinsonism without significant benefit from levodopa. VP is distinct from $\mathrm{PD}$, yet the concept of VP remains debated due to the inability of prior studies to identify specific causative changes. One reason for this may be limitations in measuring intricate BG connectivity in vivo. Given the predominant lower body parkinsonism symptoms in VP, we hypothesized that VP would be associated with decreased connectivity specifically within the BG motor loop. METHODS/STUDY POPULATION: We obtained DKI brain imaging in subjects with VP $(\mathrm{N}=7), \mathrm{PD}$ $(\mathrm{N}=21)$, and HCs $(\mathrm{N}=58)$, the latter of which had cardiovascular risk factors but no neurological symptoms. The VP and PD groups were evaluated by a parkinsonism-focused motor exam and brief cognitive testing. We compared BG motor loop connectivity between groups and investigated for correlation between connectivity and clinical scores. To account for differences in fiber counts due to the different imaging scanners and protocols between cohorts, we used a BG motor loop proportion, which was the ratio of the BG motor loop fiber count over a control loop, the visual processing pathway. We used Kruskal-Wallis rank sum test with post-hoc Dunn tests to assess imaging findings between subject groups, and Pearson's correlation to look for correlation between clinical scores and fiber counts. RESULTS/ANTICIPATED RESULTS: The whole brain connectome showed the fewest number of fibers in $\mathrm{VP}$, followed by PD, and then HC $(\mathrm{p}<0.0001)$. The BG motor loop proportion fiber count of the $\mathrm{BG}$ motor loop was lower in the VP group, compared to the PD and HC cohorts $(p=0.031)$. In the VP group, the whole brain connectome fiber count correlated with a gait and balance subscore of the Movement Disorders Society - Unified Parkinson Disease Rating Scale $(\mathrm{R}=-0.87$, $\mathrm{p}$ $=0.01$ ). DISCUSSION/SIGNIFICANCE OF IMPACT: This study indicates that VP is associated with decreased structural connectivity, with a disproportionate degree of loss in the BG motor circuitry. While the etiology for this susceptibility to injury and preferential damage to BG remains to be defined, these findings can provide an important starting point for a biological understanding of VP, and a potential future marker for diagnosis and tracking disease progression. 